\title{
A Parametrical Finite Element Formulation of the Bloch-Torrey Equation for NMR Applications
}

\author{
L. Beltrachini, Z. A. Taylor, A. F. Frangi
}

CISTIB Center for Computational Imaging \& Simulation Technologies in Biomedicine, INSIGNEO Institute for in silico Medicine, Department of Mechanical Engineering, University of Sheffield.

\section{Abstract}

We present a finite element formulation of the full Bloch-Torrey equation for nuclear magnetic resonance (NMR) applications. We obtained parametrical expressions that allow us to compute the involved matrices in a simple and fast way for any spatial convergence order. The framework here proposed is valid for many problems related to MR, as diffusion and perfusion MRI.

\section{Keywords Diffusion MRI; Finite Element Method; Nuclear Magnetic Resonance; Perfusion MRI}

\section{INTRODUCTION}

Nuclear magnetic resonance (NMR) has proven of enormous value in the investigation of porous media. Its use allows studying pore-size distributions, tortuosity, and flow permeability as a function of the relaxation time, diffusivity, and flow [1]. This information plays an important role in plenty of applications, ranging from the oil industry to medical diagnosis. A complete NMR analysis involves the solution of the Bloch-Torrey (BT) equation. However, analytically solving this equation becomes intractable for all but the simplest geometries $[1,2]$. We present an efficient numerical method to deal with arbitrarily complex domains.

\section{METHODS}

Let $\Omega=\cup_{1} \Omega_{1}$ be the domain under analysis, with $\Gamma_{\ln }=\Omega_{1} \cap$ $\Omega_{\mathrm{n}}$. Then, the BT equation becomes $[1,2]$ :

$$
\begin{gathered}
\frac{\partial \mathrm{m}_{\mathrm{l}}(\mathrm{r}, \mathrm{t})}{\partial \mathrm{t}}=\nabla \cdot\left(\mathrm{D}_{\mathrm{l}}(\mathrm{r}) \nabla \mathrm{m}_{\mathrm{l}}(\mathrm{r}, \mathrm{t})\right)-\mathrm{i} \gamma \mathrm{f}(\mathrm{t}) \mathrm{B}(\mathrm{r}) \mathrm{m}_{\mathrm{l}}(\mathrm{r}, \mathrm{t})-\frac{\mathrm{m}_{\mathrm{l}}(\mathrm{r}, \mathrm{t})}{\mathrm{T}_{\mathrm{l}}}- \\
\nabla \cdot\left(\mathrm{v}(\mathrm{r}, \mathrm{t}) \mathrm{m}_{\mathrm{l}}(\mathrm{r}, \mathrm{t})\right),\left(\mathrm{r} \in \Omega_{\mathrm{l}}\right) \quad(1) \\
\mathrm{D}_{\mathrm{l}}(\mathrm{r}) \nabla \mathrm{m}_{\mathrm{l}}(\mathrm{r}, \mathrm{t}) \cdot \mathrm{n}_{\mathrm{l}}(\mathrm{r}) \\
=\kappa_{\mathrm{ln}}\left(\mathrm{m}_{\mathrm{n}}(\mathrm{r}, \mathrm{t})-\mathrm{m}_{\mathrm{l}}(\mathrm{r}, \mathrm{t})\right),\left(\mathrm{r} \in \Gamma_{\mathrm{ln}}, \forall \mathrm{n}\right)(2) \\
\mathrm{D}_{\mathrm{l}}(\mathrm{r}) \nabla \mathrm{m}_{\mathrm{l}}(\mathrm{r}, \mathrm{t}) \cdot \mathrm{n}_{\mathrm{l}}(\mathrm{r})=-\kappa_{l}^{\mathrm{e}} \mathrm{m}_{\mathrm{l}}(\mathrm{r}, \mathrm{t}),\left(\mathrm{r} \in \Gamma_{\mathrm{l}}^{\mathrm{e}}\right), \quad(3)
\end{gathered}
$$

where $m_{1}$ is the complex transverse magnetisation we want to compute, $\Gamma_{1}^{\mathrm{e}}$ is the external boundary of $\Omega_{1}, \gamma$ is the gyromagnetic ratio of protons, $D_{1}$ is the diffusion (rank-2) tensor, $\mathrm{T}_{1}$ is the relaxation time, vis the velocity of the spins due to flow of the medium, $\mathrm{n}_{\mathrm{l}}$ is the unitary outward pointing normal to $\Omega_{1}, \kappa_{\ln }\left(\kappa_{1}^{\mathrm{e}}\right)$ is the permeability constant in $\Gamma_{\ln }\left(\Gamma_{1}^{\mathrm{e}}\right)$, and $f$ and $B$ are the effective temporal and spatial variations of the applied magnetic field. Then, $m_{l}$ is found by solving (1)-(3) with initial condition $m_{l}(r, 0)=\rho_{l}(r)$, for $t \in[0, T E]$. After obtaining $m_{l}$ we compute the complex MR signal integrating $m_{l}(r, T E) \lambda(r)$ over the domain, with $\lambda(r)$ dependent on the receiver coil [2].

To solve (1)-(3) we propose a finite element approach. To do so we discretise the problem and obtain the weak form of the variational formulation using the discontinuous Galerkin method. Discretising $\mathrm{m}_{\mathrm{l}}(\mathrm{r}, \mathrm{t})=\Sigma_{\mathrm{i}} \varphi_{\mathrm{i}}^{\mathrm{l}}(\mathrm{r}) \eta_{\mathrm{i}}^{\mathrm{l}}(\mathrm{t}), \quad$ [3] we get

$$
\begin{gathered}
\mathbf{M}_{\mathbf{l}} \frac{\partial \eta_{\mathrm{l}}}{\partial \mathrm{t}}=-\left(\mathrm{S}_{\mathrm{l}}+\mathrm{i} \gamma \mathrm{f}(\mathrm{t}) \mathrm{Q}_{\mathrm{l}}+\frac{1}{\mathrm{~T}_{\mathrm{l}}} \mathrm{M}_{\mathrm{l}}+\mathrm{J}_{\mathrm{l}}+\mathrm{\kappa}_{\mathrm{l}}^{\mathrm{e}} \mathrm{F}_{\mathrm{l}}\right) \eta_{\mathrm{l}} \\
-\sum_{\mathrm{n}} \kappa_{\mathrm{ln}} \mathrm{H}_{\mathrm{ln}} \eta_{\mathrm{n}},
\end{gathered}
$$

where:

$$
\begin{gathered}
\mathrm{M}_{\mathrm{ij}}=\int_{\Omega_{1}} \varphi_{i}^{\mathrm{l}}(\mathrm{r}) \varphi_{\mathrm{j}}^{\mathrm{l}}(\mathrm{r}) \mathrm{dr} ; \\
\mathrm{S}_{\mathrm{ij}}=\int_{\Omega_{\mathrm{l}}} \nabla \varphi_{\mathrm{j}}^{\mathrm{l}}(\mathrm{r}) \cdot \mathrm{D}_{\mathrm{l}}(\mathrm{r}) \nabla \varphi_{i}^{\mathrm{l}}(\mathrm{r}) \mathrm{dr} ; \\
\mathrm{Q}_{\mathrm{ij}}=\int_{\Omega_{\mathrm{l}}} \varphi_{\mathrm{j}}^{\mathrm{l}}(\mathrm{r}) \mathrm{B}\left(\varphi_{\mathrm{i}}^{\mathrm{l}}(\mathrm{r}), \mathrm{r}\right) \mathrm{dr} ; \\
\mathrm{J}_{\mathrm{ij}}=\int_{\Omega_{\mathrm{l}}} \varphi_{\mathrm{j}}^{\mathrm{l}}(\mathrm{r}) \nabla \cdot\left(\mathrm{v}(\mathrm{r}, \mathrm{t}) \varphi_{\mathrm{i}}^{\mathrm{l}}(\mathrm{r})\right) \mathrm{dr} ; \\
\mathrm{F}_{\mathrm{ij}}=\int_{\Gamma_{\mathrm{l}}^{\mathrm{e}}} \varphi_{\mathrm{i}}^{\mathrm{l}}(\mathrm{r}) \quad \varphi_{\mathrm{j}}^{\mathrm{l}}(\mathrm{r}) \mathrm{dr} ; \\
\mathrm{H}_{\mathrm{ij}}=\int_{\Gamma_{\mathrm{ln}}} \varphi_{\mathrm{j}}^{\mathrm{l}}(\mathrm{r})\left(\varphi_{\mathrm{i}}^{\mathrm{n}}(\mathrm{r})-\varphi_{\mathrm{i}}^{\mathrm{l}}(\mathrm{r})\right) \mathrm{dr} .
\end{gathered}
$$

To obtain numerical expressions of these matrices we transformed the integrals to the area and volume coordinate systems [3]. This method allows us to compute exact expressions as explicit functions of the mesh nodes and material properties, which is especially useful for their fast computation and to perform other parametrical experiments (e.g. as in [4]). For example, when considering first order basis functions, we find the elementary [3] mass and stiffness matrices to be $\mathrm{M}^{\mathrm{e}}=\left(1_{4}+\mathrm{I}_{4}\right) \mathrm{V}_{\mathrm{e}} / 20$ and $\mathrm{S}^{\mathrm{e}}=$ $\left(\Lambda_{\mathrm{e}}^{\mathrm{T}} \mathrm{D}_{\mathrm{e}} \Lambda_{\mathrm{e}}\right) /\left(36 \mathrm{~V}_{\mathrm{e}}\right)$ respectively, where 1 is the matrix full of ones, $\mathrm{I}$ is the identity matrix, $\Lambda$ is a $3 \times 4$ matrix depending on the elements' nodes, and e denotes the element under study. These expressions were coded in MATLAB, and will be freely available in the first's author website soon.

\section{RESULTS}

We tested the algorithm in different scenarios (different spin echo sequences, velocity fields, geometries, and physical constants), always performing as expected. In Fig. 1 we show 
a simple example where the relative error in the acquired MR signal is plotted as a function of the gradient strength (g) considering a bilayer spherical domain with radius $\left[\mathrm{r}_{1}, \mathrm{r}_{2}\right]=$ $[2,4] \mu \mathrm{m}$ (analytical solution from [2]). We considered $\kappa_{2}^{\mathrm{e}}=$ $10^{-9} \mathrm{~m} / \mathrm{s}, \mathrm{D}_{1}=\mathrm{D}_{2}=75 \times 10^{-9} \mathrm{~m}^{2} / \mathrm{s}, \mathrm{\kappa}_{12}=10^{-5} \mathrm{~m} / \mathrm{s}$, $\mathrm{T}_{1}=\mathrm{T}_{2}=\infty, \mathrm{B}$ linear, and a PGSE sequence [1] with $\delta=$ $\Delta=10 \mathrm{~ms}$. As expected, the relative error increases with $\mathrm{g}$ due to the decrease of the MR signal (from 0 to $10^{-4}$ ). The computation of the matrices is both fast and errorless.

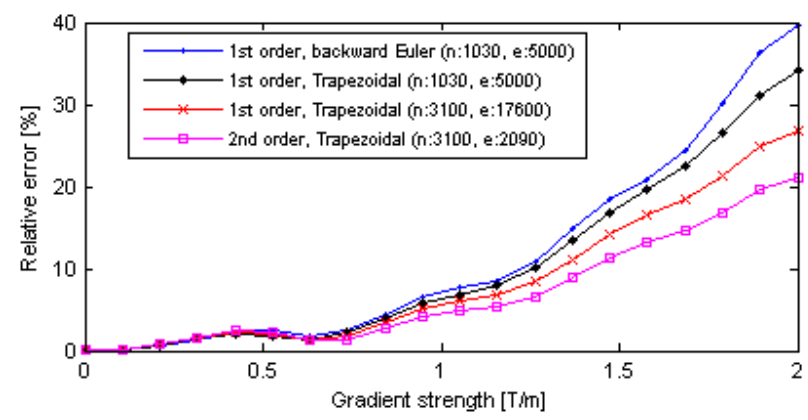

Fig.1. RE as a function of gradient strength. n:nodes, e:elements

\section{CONCLUSIONS}

We obtained an efficient and parametrical numerical solution of the complete BT equation that can be generalised to any spatial convergence order. The consideration of the flow term allows us to use this method in plenty of applications, as brain diffusion and perfusion studies. Future work will focus on using this technique to validate existing models of diffusion and perfusion signals based on gradient sensitising strategies.

\section{ACKNOWLEDGEMENTS}

This project has received funding from the European Union's Seventh Framework Programme for research, technological development and demonstration under grant agreement no FP7-ICT-2011-9-601055

\section{REFERENCES}

1. Price, NMR Studies of Transl. Motion, Cambridge, 2009.

2. Grebenkov, J Magn Res, 205: 181-195, 2010.

3. Hutton, Fundamentals of FEM, McGraw-Hill, 2004.

4. Fernández et al., Biomed Sig. Proc. Control, 8:830-837, 2013. 